\title{
ANDERSON LOCALIZATION FOR JACOBI MATRICES ASSOCIATED WITH HIGH-DIMENSIONAL SKEW SHIFTS
}

\author{
JIA SHI AND XIAOPING YUAN
}

\begin{abstract}
In this paper, we establish Anderson localization for a class of Jacobi matrices associated with skew shifts on $\mathbb{T}^{d}, d \geq 3$.
\end{abstract}

\section{INTRODUCTION AND MAIN RESULT}

Over the past thirty years, there are many papers on the topic of Anderson localization for lattice Schrödinger operators

$$
H=v_{n} \delta_{n n^{\prime}}+\Delta,
$$

where $v_{n}$ is a quasi-periodic potential, $\Delta$ is the lattice Laplacian on $\mathbb{Z}$. Anderson localization

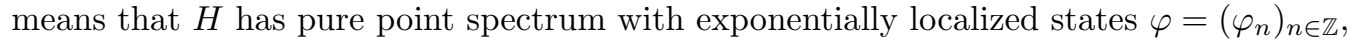

$$
\left|\varphi_{n}\right|<e^{-c|n|}, \quad|n| \rightarrow \infty .
$$

We may associate the potential $v_{n}$ to a dynamical system $T$ as follows:

$$
v_{n}=\lambda v\left(T^{n} x\right),
$$

where $v$ is real analytic on $\mathbb{T}^{d}$ and $T$ is a shift on $\mathbb{T}^{d}$ :

$$
T x=x+\omega .
$$

Fix $x=x_{0}$, if $\lambda$ is large and $\omega$ outside set of small measure, $H$ will satisfy Anderson localization.

The proof of Anderson localization is based on multi-scale analysis and semi-algebraic set theory. In this line, Bourgain and Goldstein [6] proved Anderson localization for Schrödinger operators (1.1) with help of fundamental matrix and Lyapounov exponent. By multi-scale method, Bourgain, Goldstein and Schlag [8] proved Anderson localization for Schrödinger operators on $\mathbb{Z}^{2}$

$$
H\left(\omega_{1}, \omega_{2} ; \theta_{1}, \theta_{2}\right)=\lambda v\left(\theta_{1}+n_{1} \omega_{1}, \theta_{2}+n_{2} \omega_{2}\right)+\Delta .
$$

Later, Bourgain [5] proved Anderson localization for quasi-periodic lattice Schrödinger operators on $\mathbb{Z}^{d}, d$ arbitrary. Recently, using more elaborate semi-algebraic arguments, Bourgain and Kachkovskiy [10] proved Anderson localization for two interacting quasi-periodic particles.

More generally, we can study the long range model

$$
H=v(x+n \omega) \delta_{n n^{\prime}}+S_{\phi},
$$

with $\Delta$ replaced by a Toeplitz operator

$$
S_{\phi}\left(n, n^{\prime}\right)=\hat{\phi}\left(n-n^{\prime}\right),
$$

Key words and phrases. Anderson localization, Jacobi matrices, skew shifts. 
where $\phi$ is real analytic. Bourgain [4] proved Anderson localization for the long-range quasiperiodic operators (1.6). Note that in this case, we cannot use the fundamental matrix formalism as (1.1). Bourgain's method in [4] also permits us to establish Anderson localization for band Schrödinger operators [9]

$$
H_{(n, s),\left(n^{\prime}, s^{\prime}\right)}(\omega, \theta)=\lambda v_{s}(\theta+n \omega) \delta_{n n^{\prime}} \delta_{s s^{\prime}}+\Delta,
$$

where $\left\{v_{s} \mid 1 \leq s \leq b\right\}$ are real analytic. Recently, Jian, Shi and Yuan [12] proved Anderson localization for quasi-periodic block operators with long-range interactions.

If the transformation $T$ is a skew shift on $\mathbb{T}^{2}$ :

$$
T\left(x_{1}, x_{2}\right)=\left(x_{1}+x_{2}, x_{2}+\omega\right)
$$

using transfer matrix and Lyapounov exponent, Bourgain, Goldstein and Schlag [7] proved Anderson localization for

$$
H=\lambda v\left(T^{n} x\right)+\Delta .
$$

In order to study quantum kicked rotor equation

$$
i \frac{\partial \Psi(t, x)}{\partial t}=a \frac{\partial^{2} \Psi(t, x)}{\partial x^{2}}+i b \frac{\partial \Psi(t, x)}{\partial x}+V(t, x) \Psi(t, x), \quad x \in \mathbb{T},
$$

where

$$
V(t, x)=\kappa\left(\sum_{n \in \mathbb{Z}} \delta(t-n)\right) \cos (2 \pi x),
$$

using multi-scale method, Bourgain [3] proved Anderson localization for the operator

$$
W=\phi_{m-n}\left(T^{m} x\right)
$$

where $\phi_{k}$ are trigonometric polynomials and $T$ is a skew shift on $\mathbb{T}^{2}$.

However, there are few results on high-dimensional skew shifts. When $d \geq 3$, the skew shift $T: \mathbb{T}^{d} \rightarrow \mathbb{T}^{d}$ is given by

$$
\begin{aligned}
& (T x)_{i}=x_{i}+x_{i+1}, \quad 1 \leq i \leq d-1, \\
& (T x)_{d}=x_{d}+\omega, \quad x=\left(x_{1}, \ldots, x_{d}\right) .
\end{aligned}
$$

In [13], Krüger proved positivity of Lyapounov exponents for the Schrödinger operator

$$
H=\lambda f\left(\left(T^{n} x\right)_{1}\right) \delta_{n n^{\prime}}+\Delta,
$$

where $T$ is a skew shift on $\mathbb{T}^{d}, d$ is sufficiently large, $f$ is a real, nonconstant function on $\mathbb{T}$.

In this paper, we generalized Bourgain's result on skew shifts on $\mathbb{T}^{2}[\underline{3}]$ to higher dimensional ones on $\mathbb{T}^{d}, d \geq 3$. More precisely, we consider matrices $\left(A_{m n}(x)\right)_{m, n \in \mathbb{Z}}, x \in \mathbb{T}^{d}$ associated with a skew shift $T: \mathbb{T}^{d} \rightarrow \mathbb{T}^{d}$ of the form

$$
\begin{gathered}
A_{m m}(x)=v\left(T^{m} x\right), \\
A_{m n}(x)=\phi_{m-n}\left(T^{m} x\right)+\overline{\phi_{n-m}\left(T^{n} x\right)}, \quad m \neq n,
\end{gathered}
$$

where

$$
\begin{aligned}
& v \text { is a real, nonconstant, trigonometric polynomial, } \\
& \phi_{k} \text { is a trigonometric polynomial of degree }<|k|^{C_{1}}, \\
& \qquad\left\|\phi_{k}\right\|_{\infty}<\gamma e^{-|k|} .
\end{aligned}
$$

We will prove the following result: 
Theorem 1.1. Consider a lattice operator $H_{\omega}(x)$ associated to the skew shift $T=T_{\omega}$ acting on $\mathbb{T}^{d}, d \geq 3$, of the form 1.17)-(1.21). Assume $\omega \in D C$ (diophantine condition),

$$
\|k \omega\|>c|k|^{-2}, \quad \forall k \in \mathbb{Z} \backslash\{0\} .
$$

Fix $x_{0} \in \mathbb{T}^{d}$. Then for almost all $\omega \in D C$ and $\gamma$ taken sufficiently small in (1.21), $H_{\omega}\left(x_{0}\right)$ satisfies Anderson localization.

We summarize the scheme of the proof. As mentioned above, the transfer matrix and Lyapounov exponent approach is not applicable to the long range case here. We will use the multi-scale method developed in [3], [8]. Our basic strategy is the same as that in [3], but with more complicated computations. First, we need Green's function estimates for $G_{[0, N]}(E, x)=$ $\left(R_{[0, N]}(H(x)-E) R_{[0, N]}\right)^{-1}$, where $R_{\Lambda}$ is the restriction operator to $\Lambda \subset \mathbb{Z}$. We will prove in Section 3 that

$$
\begin{gathered}
\left\|G_{[0, N]}(E, x)\right\|<e^{N^{1-}} \\
\left|G_{[0, N]}(E, x)(m, n)\right|<e^{-\frac{1}{100}|m-n|}, \quad 0 \leq m, n \leq N,|m-n|>\frac{N}{10}
\end{gathered}
$$

for $x \notin \Omega_{N}(E)$, where

$$
\operatorname{mes} \Omega_{N}(E)<e^{-N^{\sigma}}, \sigma>0 .
$$

The main difficulty here is to study the intersection of $\Omega_{N}(E)$ and skew shift orbits. We need to prove

$$
\#\left\{n=1, \ldots, M \mid T^{n} x \in \Omega_{N}(E)\right\}<M^{1-\delta}, \delta>0,
$$

where

$$
\log \log M \ll \log N \ll \log M .
$$

To obtain (1.26), we study the ergodic property of skew shifts on $\mathbb{T}^{d}$ in Section 2.

Next, in Section 4, we use decomposition of semi-algebraic set to estimate

$$
\operatorname{mes}\left[\omega \in \mathbb{T} \mid\left(\omega, T_{\omega}^{j} x\right) \in A, \exists j \sim M\right]<M^{-c}, c>0,
$$

where $x \in \mathbb{T}^{d}, A \subset \mathbb{T}^{d+1}$ is a semi-algebraic set of degree $B$ and measure $\eta$, satisfying

$$
\log B \ll \log M \ll \log \frac{1}{\eta}
$$

This is a key point to eliminate the energy $E$ in the proof of Anderson localization.

Finally, using Green's function estimates and semi-algebraic set theory, we prove Anderson localization of the operator $H_{\omega}(x)$ in Section 5 as in [6], [7].

We will use the following notations. For positive numbers $a, b, a \lesssim b$ means $C a \leq b$ for some constant $C>0 . a \ll b$ means $C$ is large. $a \sim b$ means $a \lesssim b$ and $b \lesssim a$. $N^{1-}$ means $N^{1-\epsilon}$ with some small $\epsilon>0$.

\section{An ERgOdic Property of SKeW ShIFTS ON $\mathbb{T}^{d}$}

In this section, we prove the following ergodic property of skew shifts on $\mathbb{T}^{d}$.

Lemma 2.1. Assume $\omega \in D C, T=T_{\omega}$ is the skew shift on $\mathbb{T}^{d}, \epsilon>L^{-\frac{1}{(d+1) 2^{d+1}}}$. Then

$$
\begin{array}{r}
\#\left\{n=1, \ldots, L\left\|T^{n} x-a\right\|<\epsilon\right\}<C \epsilon^{d} L, \quad C=C(d), \\
\text { where }\|x\|=\inf _{m \in \mathbb{Z}}|x-m|, x \in \mathbb{T},\|x\|=\sum_{i=1}^{d}\left\|x_{i}\right\|, x=\left(x_{1}, \ldots, x_{d}\right) \in \mathbb{T}^{d} .
\end{array}
$$


Proof. We assume $a=0$. Let $\chi$ be the indicator function of the ball $B(0, \epsilon), R=\frac{1}{\epsilon}, F_{R}$ is the Fejer kernel, then $\chi \leq C \epsilon^{d} \prod_{j=1}^{d} F_{R}\left(x_{j}\right)$.

Let $f(x)=\prod_{j=1}^{d} F_{R}\left(x_{j}\right)$, then

$$
\begin{aligned}
\sum_{n=1}^{L} \chi\left(T^{n} x\right) \leq C \epsilon^{d} \sum_{n=1}^{L} f\left(T^{n} x\right) & \leq C \epsilon^{d} \sum_{n=1}^{L} \sum_{0 \leq\left|l_{j}\right|<R} \hat{f}\left(l_{1}, \ldots, l_{d}\right) e^{2 \pi i\left\langle T^{n} x, l\right\rangle} \\
& \leq C \epsilon^{d}\left(L+\sum_{0<|k|<\frac{1}{\epsilon}} \mid \sum_{n=1}^{L} e^{\left.2 \pi i\left\langle T^{n} x, k\right\rangle \mid\right) .}\right.
\end{aligned}
$$

Let

$$
S_{k}=\left|\sum_{n=1}^{L} e^{2 \pi i\left\langle T^{n} x, k\right\rangle}\right|, \quad 0<|k|<\frac{1}{\epsilon},
$$

we only need to prove

$$
\sum_{0<|k|<\frac{1}{\epsilon}} S_{k} \leq C L
$$

From the skew shift, we have

$$
\left(T^{n} x\right)_{i}=x_{i}+n x_{i+1}+\cdots+\left(\begin{array}{c}
n \\
d-i
\end{array}\right) x_{d}+\left(\begin{array}{c}
n \\
d-i+1
\end{array}\right) \omega, \quad i=1, \ldots, d, \quad x=\left(x_{1}, \ldots, x_{d}\right) .
$$

If $k_{1}=\cdots=k_{d-1}=0$, then

$$
S_{k}=\left|\sum_{n=1}^{L} e^{2 \pi i n k_{d} \omega}\right| \leq \frac{1}{\left\|k_{d} \omega\right\|} \leq C\left|k_{d}\right|^{2} .
$$

If $k_{1}=\cdots=k_{d-2}=0, k_{d-1} \neq 0$, then $S_{k}=\left|\sum_{n=1}^{L} e^{2 \pi i f(n)}\right|$, where $f(n)=\frac{1}{2} n^{2} k_{d-1} \omega+c n, c$ is independent of $n$.

So,

$$
\begin{aligned}
S_{k}^{2} & =\left(\sum_{n=1}^{L} e^{2 \pi i f(n)}\right)\left(\sum_{n=1}^{L} e^{-2 \pi i f(n)}\right) \lesssim L+\sum_{h=1}^{L-1}\left|\sum_{n=1}^{L-h} e^{2 \pi i(f(n+h)-f(n))}\right| \\
& \lesssim L+\sum_{h=1}^{L-1} \min \left(L, \frac{1}{\left\|h k_{d-1} \omega\right\|}\right) \lesssim L+\sum_{m=1}^{\left|k_{d-1}\right| L} \min \left(L, \frac{1}{\|m \omega\|}\right) .
\end{aligned}
$$

Since $\omega \in D C$, we may find an approximant $q$ of $\omega$ satisfying

$$
L^{\frac{1}{2}}<q<L
$$

Using

we get

$$
\#\left\{M+1 \leq n \leq M+q \mid\|n \omega-u\| \leq \frac{1}{2 q}\right\} \leq 3, \quad \forall M \in \mathbb{Z}, u \in \mathbb{R}
$$

$$
\sum_{n=M+1}^{M+q} \min \left(L, \frac{1}{\|n \omega\|}\right) \lesssim L+q \log q
$$


By (2.5), (2.6), we have

$$
S_{k}^{2} \lesssim \frac{\left|k_{d-1}\right| L}{q}(L+q \log q) \lesssim\left|k_{d-1}\right| L^{\frac{3}{2}}
$$

Hence

$$
S_{k} \leq C\left|k_{d-1}\right|^{\frac{1}{2}} L^{\frac{3}{4}}
$$

If $k_{1}=\cdots=k_{d-3}=0, k_{d-2} \neq 0$, then $S_{k}=\left|\sum_{n=1}^{L} e^{2 \pi i g(n)}\right|$, where $g(n)=\frac{1}{6} n^{3} k_{d-2} \omega+b n^{2}+$ $c n, b, c$ is independent of $n$.

So,

$$
S_{k}^{2} \lesssim L+\sum_{h_{1}=1}^{L-1}\left|\sum_{n=1}^{L-h_{1}} e^{2 \pi i g_{h_{1}}(n)}\right|, \quad g_{h_{1}}(n)=g\left(n+h_{1}\right)-g(n) .
$$

We have

$$
\begin{aligned}
S_{k}^{4} & \lesssim L^{2}+L \sum_{h_{1}=1}^{L-1}\left|\sum_{n=1}^{L-h_{1}} e^{2 \pi i g_{h_{1}}(n)}\right|^{2} \\
& \lesssim L^{3}+L \sum_{h_{1}=1}^{L-1} \sum_{h_{2}=1}^{L-h_{1}-1}\left|\sum_{n=1}^{L-h_{1}-h_{2}} e^{2 \pi i\left(g_{h_{1}}\left(n+h_{2}\right)-g_{h_{1}}(n)\right)}\right| \\
& \lesssim L^{3}+L \sum_{h_{1}=1}^{L} \sum_{h_{2}=1}^{L} \min \left(L, \frac{1}{\left\|h_{1} h_{2} k_{d-2} \omega\right\|}\right) .
\end{aligned}
$$

Using

$$
\#\left\{\left(h_{1}, h_{2}\right) \in \mathbb{Z}^{2} \mid h_{1} h_{2}=N\right\} \lesssim N^{0+}
$$

we get

$$
S_{k}^{4} \lesssim L^{3}+L^{1+} \sum_{m=1}^{\left|k_{d-2}\right| L^{2}} \min \left(L, \frac{1}{\|m \omega\|}\right) \lesssim L^{3}+L^{1+} \frac{\left|k_{d-2}\right| L^{2}}{q}(L+q \log q) \lesssim\left|k_{d-2}\right| L^{\frac{7}{2}+} .
$$

Hence

$$
S_{k} \leq C\left|k_{d-2}\right|^{\frac{1}{4}} L^{\frac{7}{8}+} .
$$

Repeat the argument above, we get

$$
S_{k} \leq C\left|k_{d-j}\right|^{\frac{1}{2 j}} L^{1-\frac{1}{2^{j+1}}+}, \quad k_{1}=\cdots=k_{d-j-1}=0, k_{d-j} \neq 0, \quad 2 \leq j \leq d-1 .
$$

By (2.4), (2.7), (2.9), we have

$$
\begin{aligned}
\sum_{0<|k|<\frac{1}{\epsilon}} S_{k} & \lesssim \sum_{\left|k_{d}\right|<\frac{1}{\epsilon}}\left|k_{d}\right|^{2}+\frac{1}{\epsilon} \sum_{\left|k_{d-1}\right|<\frac{1}{\epsilon}}\left|k_{d-1}\right|^{\frac{1}{2}} L^{\frac{3}{4}}+\sum_{j=2}^{d-1} \frac{1}{\epsilon^{j}}\left(\sum_{\left|k_{d-j}\right|<\frac{1}{\epsilon}}\left|k_{d-j}\right|^{\frac{1}{2^{j}}} L^{1-\frac{1}{2^{j+1}}+}\right) \\
& \lesssim\left(\frac{1}{\epsilon}\right)^{3}+\frac{1}{\epsilon}\left(\frac{1}{\epsilon}\right)^{\frac{3}{2}} L^{\frac{3}{4}}+\sum_{j=2}^{d-1}\left(\left(\frac{1}{\epsilon}\right)^{\frac{1}{2^{j}}+j+1} L^{1-\frac{1}{2^{j}+1}+}\right) \lesssim L .
\end{aligned}
$$

This proves (2.2) and Lemma 2.1.

Remark 2.2. In the proof of Lemma [2.1, we only need to assume

$$
\|k \omega\|>c|k|^{-2}, \quad \forall 0<|k| \leq L .
$$




\section{Green's FunCtion estimates} [3].

In this section, we will prove the Green's function estimates using multi-scale analysis in

We need the following lemma.

Lemma 3.1 (Lemma 3.16 in [3]). Let $A(x)=\left\{A_{m n}(x)\right\}_{1 \leq m, n \leq N}$ be a matrix valued function on $\mathbb{T}^{d}$ such that

$$
A(x) \text { is self adjoint for } x \in \mathbb{T}^{d} \text {, }
$$

$$
A_{m n}(x) \text { is a trigonometric polynomial of degree }<N^{C_{1}} \text {, }
$$

where $c_{2}, C_{1}, C_{2}>0$ are constants.

Let $0<\delta<1$ be sufficiently small, $M=N^{\delta^{6}}, L_{0}=N^{\frac{1}{100}} \delta^{2}, 0<c_{3}<\frac{1}{10} c_{2}$.

Assume that for any interval $I \subset[1, N]$ of size $L_{0}$, except for $x$ in a set of measure at most $e^{-L_{0}^{\delta^{3}}}$

$$
\begin{gathered}
\left\|\left(R_{I} A(x) R_{I}\right)^{-1}\right\|<e^{L_{0}^{1-}} \\
\left|\left(R_{I} A(x) R_{I}\right)^{-1}(m, n)\right|<e^{-c_{3}|m-n|}, \quad m, n \in I,|m-n|>\frac{L_{0}}{10} .
\end{gathered}
$$

Fix $x \in \mathbb{T}^{d}, n_{0} \in[1, N]$ is called a good site if $I_{0}=\left[n_{0}-\frac{M}{2}, n_{0}+\frac{M}{2}\right] \subset[1, N]$,

$$
\begin{gathered}
\left\|\left(R_{I_{0}} A(x) R_{I_{0}}\right)^{-1}\right\|<e^{M^{1-}} \\
\left|\left(R_{I_{0}} A(x) R_{I_{0}}\right)^{-1}(m, n)\right|<e^{-c_{3}|m-n|}, \quad m, n \in I_{0},|m-n|>\frac{M}{10} .
\end{gathered}
$$

Denote $\Omega(x) \subset[1, N]$ the set of bad sites. Assume that for any interval $J \subset[1, N],|J|>N^{\frac{\delta}{5}}$, we have $|J \cap \Omega(x)|<|J|^{1-\delta}$.

Then

$$
\begin{gathered}
\left\|A(x)^{-1}\right\|<e^{N^{1-\frac{\delta}{C(d)}}}, \\
\left|A(x)^{-1}(m, n)\right|<e^{-c_{3}^{\prime}|m-n|}, \quad m, n \in[1, N],|m-n|>\frac{N}{10}
\end{gathered}
$$

except for $x$ in a set of measure at most $e^{-\frac{N^{\delta^{2}}}{C(d)}}$, where $C(d)$ is a constant depending on $d$, $c_{3}^{\prime}>c_{3}-(\log N)^{-8}$.

By Lemma 2.1. Lemma 3.1, we can prove the Green's function estimates.

Proposition 3.2. Let $T=T_{\omega}: \mathbb{T}^{d} \rightarrow \mathbb{T}^{d}$ be the skew shift with frequency $\omega$ satisfying

$$
\|k \omega\|>c|k|^{-2}, \quad \forall 0<|k| \leq N .
$$

$A_{m n}(x)$ is the form (1.17)-(1.21), $\gamma$ in (1.21) is small.

Then for all $N$ and energy $E$,

$$
\begin{gathered}
\left\|G_{[0, N]}(E, x)\right\|<e^{N^{1-}} \\
\left|G_{[0, N]}(E, x)(m, n)\right|<e^{-\frac{1}{100}|m-n|}, \quad 0 \leq m, n \leq N,|m-n|>\frac{N}{10}
\end{gathered}
$$


for $x \notin \Omega_{N}(E)$, where

$$
\operatorname{mes} \Omega_{N}(E)<e^{-N^{\sigma}}, \sigma>0 .
$$

Proof. Since $T^{n}\left(x_{1}, \ldots, x_{d}\right)=\left(x_{1}+n x_{2}+\cdots+\left(\begin{array}{c}n \\ d-1\end{array}\right) x_{d}+\left(\begin{array}{c}n \\ d\end{array}\right) \omega, \ldots, x_{d}+n \omega\right), A_{m n}(x)$ is a trigonometric polynomial in $x$ of degree $<(|m|+|n|)^{C_{1}+d},\left\{A_{m n}(x)-E\right\}_{0 \leq m, n \leq N}$ satisfy (3.1)-(3.3) with $c_{2}=1, C_{2}=\gamma$.

First fix any large initial scale $N_{0}$ and choose $\gamma=\gamma\left(N_{0}\right)$ small, using Lojasiewicz's inequality (see Section 4 in [3] ), we get

$$
\left|G_{\left[0, N_{0}\right]}(E, x)(m, n)\right|<e^{N_{0}^{\frac{1}{2}}-\frac{1}{2}|m-n|}, \quad 0 \leq m, n \leq N_{0}
$$

except for $x$ in a set of measure $<e^{-c N_{0}^{\frac{1}{2}}}$.

Then we estabish inductively on the scale $N$ that

$$
\text { mes }\left[x \in \mathbb{T}^{d}|| G_{[0, N]}(E, x)(m, n) \mid>e^{N^{1-}-c_{3}|m-n| \chi_{|m-n|>\frac{N}{10}}}, \exists 0 \leq m, n \leq N\right]<e^{-N^{\delta^{3}}},
$$

where $c_{3}>\frac{1}{100}, 0<\delta<1$ is a fixed small number.

(3.14) implies (3.15) for an initial large scale $N_{0}$.

Assume (3.15) holds up to scale $L_{0}=N^{\frac{1}{100}} \delta^{2}$. Since $A_{m+1, n+1}(x)=A_{m n}(T x)$, we have

$$
R_{I}(A(x)-E) R_{I}=R_{[0, N]}\left(A\left(T^{n} x\right)-E\right) R_{[0, N]}, G_{I}(E, x)=G_{[0, N]}\left(E, T^{n} x\right), \quad I=[n, n+N] .
$$

Since $T$ is measure preserving, (3.4), (3.5) will hold for $x$ outside a set of measure at most $e^{-L_{0}^{\delta^{3}}}$. Denote $\Omega(x) \subset[0, N]$ the set of bad sites with respect to scale $M=N^{\delta^{6}} . n_{0} \notin \Omega(x)$ means

$$
\begin{aligned}
& \left|G_{[0, M]}\left(E, T^{n_{0}-\frac{M}{2}} x\right)(m, n)\right|= \\
& \left|G_{\left[n_{0}-\frac{M}{2}, n_{0}+\frac{M}{2}\right]}(E, x)\left(m+n_{0}-\frac{M}{2}, n+n_{0}-\frac{M}{2}\right)\right|<e^{M^{1-}-c_{3}|m-n| \chi_{|m-n|>\frac{M}{10}}} .
\end{aligned}
$$

From the inductive hypothesis, we have

$$
\left|G_{[0, M]}(E, x)(m, n)\right|<e^{M^{1-}-c_{3}|m-n| \chi_{|m-n|>\frac{M}{10}}}, 0 \leq m, n \leq M, \quad \forall x \notin \Omega, \quad \operatorname{mes} \Omega<e^{-M^{\delta^{3}}} .
$$

By (3.16), (3.17), Lemma 3.1, we only need to show that for any $x \in \mathbb{T}^{d}, N^{\frac{\delta}{5}}<L<N$,

$$
\#\left\{1 \leq n \leq L \mid T^{n} x \in \Omega\right\}<L^{1-\delta} .
$$

Since $A_{m n}(x)$ is a trigonometric polynomial of degree $<(|m|+|n|)^{C}$, we can express $G_{[0, M]}(E, x)(m, n)$ as a ratio of determinants to write (3.17) in the form

$$
P_{m n}\left(\cos x_{1}, \sin x_{1}, \ldots, \cos x_{d}, \sin x_{d}\right) \leq 0,
$$

where $P_{m n}$ is a polynomial of degree at most $M^{C}$. Replacing cos, sin by truncated power series, permits us to replace (3.19) by

$$
P_{m n}\left(x_{1}, \ldots, x_{d}\right) \leq 0, \quad \operatorname{deg} P_{m n}<M^{C} .
$$

So, $\Omega$ may be viewed as a semi-algebraic set of degree at most $M^{C}$. (For properties of semialgebraic sets, see Section 4.) When $\epsilon>e^{-\frac{1}{d} M^{\delta^{3}}}$, by Corollary 4.4 $\Omega$ may be covered by at 
most $M^{C}\left(\frac{1}{\epsilon}\right)^{d-1} \epsilon$-balls. Choosing $\epsilon=L^{-\frac{1}{(d+1) 2^{d+1}}}>N^{-1}>e^{-\frac{1}{d} M^{\delta^{3}}}$, by (3.10), using Lemma 2.1, Remark 2.2, we have

$$
\#\left\{1 \leq n \leq L \mid T^{n} x \in \Omega\right\}<M^{C}\left(\frac{1}{\epsilon}\right)^{d-1} \epsilon^{d} L<L^{C \delta^{5}+1-\frac{1}{(d+1) 2^{d+1}}}<L^{1-\delta},
$$

when $\delta$ is small enough.

This proves (3.18) and Proposition 3.2

\section{Semi-Algebraic Sets}

We recall some basic facts of semi-algebraic sets. Let $\mathcal{P}=\left\{P_{1}, \ldots, P_{s}\right\} \subset \mathbb{R}\left[X_{1}, \ldots, X_{n}\right]$ be a family of real polynomials whose degrees are bounded by $d$. A semi-algebraic set is given by

$$
S=\bigcup_{j} \bigcap_{l \in L_{j}}\left\{\mathbb{R}^{n} \mid P_{l} s_{j l} 0\right\},
$$

where $L_{j} \subset\{1, \ldots, s\}, s_{j l} \in\{\leq, \geq,=\}$ are arbitrary. We say that $S$ has degree at most $s d$ and its degree is the inf of $s d$ over all representations as in (4.1).

The projection of a semi-algebraic set of $\mathbb{R}^{n}$ onto $\mathbb{R}^{m}$ is semi-algebraic.

Proposition $4.1([2])$. Let $S \subset \mathbb{R}^{n}$ be a semi-algebraic set of degree $B$, then any projection of $S$ has degree at most $B^{C}, C=C(n)$.

We need the following bound on the number of connected components.

Proposition $4.2([1])$. Let $S \subset \mathbb{R}^{n}$ be a semi-algebraic set of degree $B$, then the number of connected components of $S$ is bounded by $B^{C}, C=C(n)$.

A more advanced part of the theory of semi-algebraic sets is the following triangulation theorem.

Theorem 4.3 (11]). For any positive integers $r, n$, there exists a constant $C=C(n, r)$ with the following property: Any semi-algebraic set $S \subset[0,1]^{n}$ can be triangulated into $N \lesssim(\operatorname{deg} S+1)^{C}$ simplices, where for every closed $k$-simplex $\Delta \subset S$, there exists a homeomorphism $h_{\Delta}$ of the regular simplex $\Delta^{k} \subset \mathbb{R}^{k}$ with unit edge length onto $\Delta$ such that $\left\|D_{r} h_{\Delta}\right\| \leq 1$.

Corollary 4.4 (Corollary 9.6 in [4]). Let $S \subset[0,1]^{n}$ be semi-algebraic of degree B. Let $\epsilon>0, \operatorname{mes}_{n} S<\epsilon^{n}$. Then $S$ may be covered by at most $B^{C}\left(\frac{1}{\epsilon}\right)^{n-1} \epsilon$-balls.

Finally, we will make essential use of the following transversality property.

Lemma $4.5((1.5)$ in $[\underline{5}])$. Let $S \subset[0,1]^{n=n_{1}+n_{2}}$ be a semi-algebraic set of degree $B$ and

$$
\operatorname{mes}_{n} S<\eta, \quad \log B \ll \log \frac{1}{\eta}, \quad \epsilon>\eta^{\frac{1}{n}},
$$

denote $(x, y) \in[0,1]^{n_{1}} \times[0,1]^{n_{2}}$ the product variable.

Then there is a decomposition $S=S_{1} \cup S_{2}, S_{1}$ satisfying

$$
\operatorname{mes}_{n_{1}}\left(\operatorname{Proj}_{x} S_{1}\right)<B^{C} \epsilon
$$

and $S_{2}$ satisfying the transversality property

$$
\operatorname{mes}_{n_{2}}\left(S_{2} \cap L\right)<B^{C} \epsilon^{-1} \eta^{\frac{1}{n}}
$$

for any $n_{2}$-dimensional hyperplane $L$ such that $\max _{1 \leq j \leq n_{1}}\left|\operatorname{Proj}_{L}\left(e_{j}\right)\right|<\frac{\epsilon}{100}$, where $\left\{e_{j} \mid 1 \leq j \leq\right.$ $\left.n_{1}\right\}$ are $x$-coordinate vectors.

Now we can prove the following lemma. 
Lemma 4.6. Let $S \subset[0,1]^{d+1}$ be a semi-algebraic set of degree $B$ such that

$$
\operatorname{mes} S<e^{-B^{\sigma}}, \sigma>0 \text {. }
$$

Let $M$ satisfy

$$
\log \log M \ll \log B \ll \log M .
$$

Then for all $x \in \mathbb{T}^{d}$,

$$
\operatorname{mes}\left[\omega \in \mathbb{T} \mid\left(\omega, T_{\omega}^{j} x\right) \in S, \exists j \sim M\right]<M^{-c}, c>0 .
$$

Proof. For $x^{0}=\left(x_{1}^{0}, \ldots, x_{d}^{0}\right) \in \mathbb{T}^{d}$, we study the intersection of $S \subset[0,1]^{d+1}$ and sets

$$
\left\{\left(\omega, x_{1}, \ldots, x_{d}\right) \mid \omega \in[0,1]\right\},
$$

where $x_{i}=\left(T_{\omega}^{j} x^{0}\right)_{i}=x_{i}^{0}+j x_{i+1}^{0}+\cdots+\left(\begin{array}{c}j \\ d-i\end{array}\right) x_{d}^{0}+\left(\begin{array}{c}j \\ d-i+1\end{array}\right) \omega, 1 \leq i \leq d$ are considered (mod 1).

By (4.5), (4.6), we have

$$
\operatorname{mes}_{d+1} S<\eta=e^{-B^{\sigma}}, \quad \log B \ll \log M \ll \log \frac{1}{\eta} .
$$

Take $\epsilon=M^{-1+}$ and apply Lemma 4.5. $S=S_{1} \cup S_{2}$. Since $\operatorname{mes}_{1}\left(\operatorname{Proj}_{\omega} S_{1}\right)<B^{C} M^{-1+}=$ $M^{-1+}$, restriction of $\omega$ permits us to replace $S$ by $S_{2}$ satisfying

$$
\operatorname{mes}_{d}\left(S_{2} \cap L\right)<B^{C} \epsilon^{-1} \eta^{\frac{1}{d+1}}<\eta^{\frac{1}{d+2}},
$$

whenever $L$ is a $d$-dimensional hyperplane satisfying $\left|\operatorname{Proj}_{L}\left(e_{0}\right)\right|<\frac{\epsilon}{100}$, where $e_{0}$ is the $\omega$ coordinate vector.

Fixing $j$,(4.8) considered as subset of $[0,1]^{d+1}$ lies in the union of the parallel $d$-dimensional hyperplanes

$$
Q_{m_{1}}^{(j)}=\left[\omega=\frac{x_{d}}{j}\right]-\frac{m_{1}+x_{d}^{0}}{j} e_{0}, \quad\left|m_{1}\right|<M .
$$

By (4.10), we have

$$
\operatorname{mes}_{d}\left(S \cap Q_{m_{1}}\right)<\eta^{\frac{1}{d+2}} .
$$

Fixing $m_{1}$, consider the semi-algebraic set $S \cap Q_{m_{1}}$ and its intersection with the parallel $(d-1)$-dimensional hyperplanes

$$
Q_{m_{1}, m_{2}}^{(j)}=Q_{m_{1}} \cap\left[x_{d}=\frac{2}{j-1} x_{d-1}-\frac{2}{j-1}\left(x_{d-1}^{0}+\frac{j+1}{2} x_{d}^{0}+m_{2}\right)\right], \quad\left|m_{2}\right|<M .
$$

Take $\epsilon=M^{-1+}$ and apply Lemma 4.5] in $Q_{m_{1}}, S \cap Q_{m_{1}}=S_{m_{1}}^{1} \cup S_{m_{1}}^{2}$, where

$$
\operatorname{Proj}_{x_{d}} S_{m_{1}}^{1} \text { is a union of at most } B^{C} \text { intervals of measure at most } B^{C} M^{-1+} \text {, }
$$
and by (4.12), we have

$$
\operatorname{mes}_{d-1}\left(S_{m_{1}}^{2} \cap Q_{m_{1}, m_{2}}\right)<B^{C} M \eta^{\frac{1}{d(d+2)}}<\eta^{\frac{1}{(d+2)^{2}}} .
$$

Fixing $m_{2}$, consider the semi-algebraic set $S_{m_{1}}^{2} \cap Q_{m_{1}, m_{2}}$ and its intersection with the parallel $(d-2)$-dimensional hyperplanes

$$
Q_{m_{1}, m_{2}, m_{3}}^{(j)}=Q_{m_{1}, m_{2}} \cap\left[x_{d-1}=\frac{3}{j-2} x_{d-2}-\frac{3}{j-2}\left(x_{d-2}^{0}+\cdots+\frac{j(j+1)}{6} x_{d}^{0}+m_{3}\right)\right],
$$

where $\left|m_{3}\right|<M$. 
Take $\epsilon=M^{-1+}$ and apply Lemma 4.5 in $Q_{m_{1}, m_{2}}, S_{m_{1}}^{2} \cap Q_{m_{1}, m_{2}}=S_{m_{1}, m_{2}}^{1} \cup S_{m_{1}, m_{2}}^{2}$, where $\operatorname{Proj}_{x_{d-1}} S_{m_{1}, m_{2}}^{1}$ is a union of at most $B^{C}$ intervals of measure at most $B^{C} M^{-1+}$, and by (4.15), we have

$$
\operatorname{mes}_{d-2}\left(S_{m_{1}, m_{2}}^{2} \cap Q_{m_{1}, m_{2}, m_{3}}\right)<\eta^{\frac{1}{(d+2)^{3}}} .
$$

Repeat the argument above, fixing $m_{i}, 2 \leq i \leq d-1$, consider the semi-algebraic set $S_{m_{1}, \ldots, m_{i-1}}^{2} \cap Q_{m_{1}, \ldots, m_{i}}$ and its intersection with the parallel $(d-i)$-dimensional hyperplanes

$Q_{m_{1}, \ldots, m_{i+1}}^{(j)}=Q_{m_{1}, \ldots, m_{i}} \cap\left[x_{d-i+1}=\frac{i+1}{j-i} x_{d-i}-\frac{i+1}{j-i}\left(x_{d-i}^{0}+\cdots+\frac{1}{i+1}\left(\begin{array}{c}j+1 \\ i\end{array}\right) x_{d}^{0}+m_{i+1}\right)\right]$,

where $\left|m_{i+1}\right|<M$.

Take $\epsilon=M^{-1+}$ and apply Lemma 4.5 in $Q_{m_{1}, \ldots, m_{i}}, S_{m_{1}, \ldots, m_{i-1}}^{2} \cap Q_{m_{1}, \ldots, m_{i}}=S_{m_{1}, \ldots, m_{i}}^{1} \cup$ $S_{m_{1}, \ldots, m_{i}}^{2}$, where

$\operatorname{Proj}_{x_{d-i+1}} S_{m_{1}, \ldots, m_{i}}^{1}$ is a union of at most $B^{C}$ intervals of measure at most $B^{C} M^{-1+}$, and

$$
\operatorname{mes}_{d-i}\left(S_{m_{1}, \ldots, m_{i}}^{2} \cap Q_{m_{1}, \ldots, m_{i+1}}\right)<\eta^{\frac{1}{(d+2)^{i+1}}} .
$$

Finally, fixing $m_{d-1}$, consider the semi-algebraic set $S_{m_{1}, \ldots, m_{d-2}}^{2} \cap Q_{m_{1}, \ldots, m_{d-1}}$ and its intersection with the parallel lines

$Q_{m_{1}, \ldots, m_{d}}^{(j)}=Q_{m_{1}, \ldots, m_{d-1}} \cap\left[x_{2}=\frac{d}{j-d+1} x_{1}-\frac{d}{j-d+1}\left(x_{1}^{0}+\cdots+\frac{1}{d}\left(\begin{array}{l}j+1 \\ d-1\end{array}\right) x_{d}^{0}+m_{d}\right)\right]$,

where $\left|m_{d}\right|<M$.

Take $\epsilon=M^{-1+}$ and apply Lemma4.5 in $Q_{m_{1}, \ldots, m_{d-1}}, S_{m_{1}, \ldots, m_{d-2}}^{2} \cap Q_{m_{1}, \ldots, m_{d-1}}=S_{m_{1}, \ldots, m_{d-1}}^{1} \cup$ $S_{m_{1}, \ldots, m_{d-1}}^{2}$, where

(4.20) $\operatorname{Proj}_{x_{2}} S_{m_{1}, \ldots, m_{d-1}}^{1}$ is a union of at most $B^{C}$ intervals of measure at most $B^{C} M^{-1+}$, and

$$
\operatorname{mes}_{1}\left(S_{m_{1}, \ldots, m_{d-1}}^{2} \cap Q_{m_{1}, \ldots, m_{d}}\right)<\eta^{\frac{1}{(d+2)^{d}}} .
$$

Summing (4.21) over $j, m_{1}, \ldots, m_{d}$, the collected contribution in the $\omega$-parameter is less than $M^{-d} M^{d+1} B^{C} M \eta^{\frac{1}{(d+2)^{d}}}<\eta^{\frac{1}{(d+2)^{d+1}}}$. So, we only need to consider the contribution of $S_{m_{1}, \ldots, m_{i}}^{1}$ (4.17). We just deal with $S_{m_{1}, \ldots, m_{d-1}}^{1}$ below, since for other sets, the method is similar.

If (4.7) fails, we have

$$
\begin{gathered}
\sum_{j \sim M,\left|m_{1}\right|, \ldots,\left|m_{d}\right|<M} \operatorname{mes}\left[\operatorname{Proj}_{\omega} \operatorname{Proj}_{x_{2}}\left(S_{m_{1}, \ldots, m_{d-1}}^{1} \cap Q_{m_{1}, \ldots, m_{d}}^{(j)}\right)\right]>M^{0-}, \\
\sum_{j \sim M,\left|m_{1}\right|, \ldots,\left|m_{d}\right|<M} \operatorname{mes}\left[\operatorname{Proj}_{x_{2}}\left(S_{m_{1}, \ldots, m_{d-1}}^{1} \cap Q_{m_{1}, \ldots, m_{d}}^{(j)}\right)\right]>M^{d-1-} .
\end{gathered}
$$

So, there is a set $J \subset \mathbb{Z} \cap[j \sim M],|J|>M^{1-}$ such that for each $j \in J$, there are at least $M^{d-1-}$ values of $\left(m_{1}, \ldots, m_{d-1}\right)$ satisfying

$$
\sum_{\left|m_{d}\right|<M} \operatorname{mes}\left[\operatorname{Proj}_{x_{2}}\left(S_{m_{1}, \ldots, m_{d-1}}^{1} \cap Q_{m_{1}, \ldots, m_{d}}^{(j)}\right)\right]>M^{-1} .
$$


By (4.20), $S_{m_{1}, \ldots, m_{d-1}}^{1} \cap Q_{m_{1}, \ldots, m_{d}}^{(j)} \neq \emptyset$ for at most $M^{0+}$ values of $m_{d}$. Hence

$$
\max _{m_{d}} \operatorname{mes}_{1}\left(S \cap Q_{m_{1}, \ldots, m_{d}}^{(j)}\right)>M^{0-} .
$$

For fixed $j$,

$$
Q_{m_{1}, \ldots, m_{d}}^{(j)} / / \xi_{j} / /\left(1,\left(\begin{array}{l}
j \\
d
\end{array}\right), \ldots, j\right)^{T}, \quad\left\|\xi_{j}\right\|=1
$$

Denote $S_{x}$ the intersection of $S$ and the $d$-dimensional hyperplane $\left[x^{\prime}=x\right]$. From (4.24), to each $\left(m_{1}, \ldots, m_{d-1}\right)$ we can associate some $m_{d}$, such that

$$
\int_{0}^{1} \#\left\{\left|m_{1}\right|, \ldots,\left|m_{d-1}\right|<M \mid S_{x} \cap Q_{m_{1}, \ldots, m_{d}} \neq \emptyset\right\} d x>M^{d-1-} .
$$

If $\operatorname{mes}_{d} S_{x}<\eta^{\frac{1}{2}}$, then $S_{x} \cap Q_{m_{1}, \ldots, m_{d}} \neq \emptyset$ implies $\operatorname{dist}\left(Q_{m_{1}, \ldots, m_{d}}, \partial S_{x}\right)<\eta^{\frac{1}{2 d}}$, where $\partial S_{x}$ is a union of at most $B^{C}$ connected $(d-1)$-dimensional algebraic set of degree at most $B^{C}$. From (4.26), it follows that there is a fixed $(d-1)$-dimensional algebraic set $\Gamma=\Gamma^{(j)}$ of degree at most $B^{C}$ such that for $x \in[0,1]$ in a set of measure $>M^{0-}$, there are at least $M^{d-1-} \frac{1}{M}$-separated points that are $\eta^{\frac{1}{2 d}}$-close to both $\partial S_{x}$ and $\Gamma+x \xi_{j}$. Hence $\left(\Gamma+x \xi_{j}\right) \cap S_{\eta_{1}}\left(\eta_{1}\right.$-neighborhood of $\left.S, \eta_{1}=2 \eta^{\frac{1}{2 d}}\right)$ contains at least $M^{d-1-} \frac{1}{M}$-separated points. So, $\operatorname{mes}_{d-1}\left(\left(\Gamma+x \xi_{j}\right) \cap S_{\eta_{1}}\right)>M^{0-}$.

The hypercylinder $\mathcal{C}^{(j)}=t \xi_{j}+\Gamma^{(j)}$ satisfies

$$
\operatorname{mes}_{d}\left(\mathcal{C}^{(j)} \cap S_{\eta_{1}}\right)>M^{0-} .
$$

By Corollary 4.4, we have

$$
\operatorname{mes}_{d+1} S_{\eta_{1}}<B^{C} \eta_{1} \text {. }
$$

Since (4.27) holds for all $j \in J$, by (4.27), (4.28), we have

$$
\sum_{j_{1}, \ldots, j_{d+1} \in J} \operatorname{mes}_{d+1}\left[\bigcap_{1 \leq i \leq d+1} \mathcal{C}_{\eta_{1}}^{\left(j_{i}\right)}\right]>\eta_{1} M^{d+1-} .
$$

So, there are distinct $j_{1}, \ldots, j_{d+1} \sim M$ such that

$$
\operatorname{mes}_{d+1}\left[\bigcap_{1 \leq i \leq d+1} \mathcal{C}_{\eta_{1}}^{\left(j_{i}\right)}\right]>\eta_{1} M^{0-} .
$$

By (4.25), using Vandermonde determinant, we have

$$
\operatorname{det}\left[\xi_{j_{1}}, \ldots, \xi_{j_{d+1}}\right] \neq 0,
$$

for distinct $j_{1}, \ldots, j_{d+1}$. So, the vectors $\xi_{j_{1}}, \ldots, \xi_{j_{d+1}}$ are not in any $d$-dimensional hyperplane. Since $\log M \ll \log \frac{1}{\eta_{1}}$, this leads to a contradiction with (4.29).

This proves Lemma 4.6

\section{Proof of Anderson localization}

In this section, we give the proof of Anderson localization as in [6].

By application of the resolvent identity, we have the following

Lemma 5.1 (Lemma 10.33 in [4]). Let $I \subset \mathbb{Z}$ be an interval of size $N$ and $\left\{I_{\alpha}\right\}$ be subintervals of size $M \ll N$. Assume that

(i) If $k \in I$, then there is some $\alpha$ such that $\left[k-\frac{M}{4}, k+\frac{M}{4}\right] \cap I \subset I_{\alpha}$.

(ii) For all $\alpha$,

$$
\left\|G_{I_{\alpha}}\right\|<e^{M^{1-}},\left|G_{I_{\alpha}}\left(n_{1}, n_{2}\right)\right|<e^{-c_{0}\left|n_{1}-n_{2}\right|}, n_{1}, n_{2} \in I_{\alpha},\left|n_{1}-n_{2}\right|>\frac{M}{10} .
$$


Then

$$
\left|G_{I}\left(n_{1}, n_{2}\right)\right|<e^{-\left(c_{0}-\right)\left|n_{1}-n_{2}\right|}, n_{1}, n_{2} \in I,\left|n_{1}-n_{2}\right|>\frac{N}{10} .
$$

Let $T=T_{\omega}$ be the skew shift on $\mathbb{T}^{d}$ with frequency $\omega$ satisfying

$$
\|k \omega\|>c|k|^{-2}, \quad \forall k \in \mathbb{Z} \backslash\{0\} .
$$

Fix $x_{0} \in \mathbb{T}^{d}$.

$$
\begin{gathered}
H\left(x_{0}\right)(m, m)=v\left(T^{m} x_{0}\right), \\
H\left(x_{0}\right)(m, n)=\phi_{m-n}\left(T^{m} x_{0}\right)+\overline{\phi_{n-m}\left(T^{n} x_{0}\right)}, \quad m \neq n
\end{gathered}
$$

with $v$ and $\phi_{k}$ satisfying (1.19)-(1.21) and $\gamma$ taken small enough. Then we have

Theorem 5.2. For almost all $\omega$ satisfying (5.1), the lattice operator $H_{\omega}\left(x_{0}\right)$ satisfies Anderson localization.

Proof. To establish Anderson localization, it suffices to show that if $\xi=\left(\xi_{n}\right)_{n \in \mathbb{Z}}, E \in \mathbb{R}$ satisfy

$$
\begin{gathered}
\left|\xi_{n}\right|<C|n|, \quad|n| \rightarrow \infty, \\
H\left(x_{0}\right) \xi=E \xi
\end{gathered}
$$

then

$$
\left|\xi_{n}\right|<e^{-c|n|}, \quad|n| \rightarrow \infty .
$$

Let $M=N^{C_{0}}, L=M^{C}$. Denote $\Omega \subset \mathbb{T}^{d}$ the set of $x$ such that

$$
\left|G_{[-M, M]}(E, x)(m, n)\right|<e^{M^{1-}-\frac{1}{100}|m-n| \chi_{|m-n|>\frac{M}{10}}}
$$

fails for some $|m|,|n| \leq M$. It was shown in Section 3 that

$$
\#\left\{1 \leq|n| \leq L \mid T^{n} x_{0} \in \Omega\right\}<L^{1-\delta} .
$$

So, we may find an interval $I \subset[0, L]$ of size $M$, such that

$$
T^{n_{0}} x_{0} \notin \Omega, \quad \forall n_{0} \in I \cup(-I) .
$$

Hence

$$
\left|G_{\left[n_{0}-M, n_{0}+M\right]}\left(E, x_{0}\right)(m, n)\right|<e^{M^{1-}-\frac{1}{100}|m-n| \chi_{|m-n|>\frac{M}{10}}}, m, n \in\left[n_{0}-M, n_{0}+M\right] .
$$

By (5.4), (5.5), (5.7), we have

$$
\left|\xi_{n_{0}}\right| \leq \sum_{n^{\prime} \in\left[n_{0}-M, n_{0}+M\right], n^{\prime \prime} \notin\left[n_{0}-M, n_{0}+M\right]} e^{M^{1-}-\frac{1}{100}\left|n_{0}-n^{\prime}\right| \chi_{\left|n_{0}-n^{\prime}\right|>\frac{M}{10}}} e^{-\left|n^{\prime}-n^{\prime \prime}\right|}\left|\xi_{n^{\prime \prime}}\right|<e^{-\frac{M}{200}} .
$$

Denoting $j_{0}$ the center of $I$, we have

$$
1=\left|\xi_{0}\right| \leq\left\|G_{\left[-j_{0}, j_{0}\right]}\left(x_{0}, E\right)\right\|\left\|R_{\left[-j_{0}, j_{0}\right]} H\left(x_{0}\right) R_{\mathbb{Z} \backslash\left[-j_{0}, j_{0}\right]} \xi\right\| .
$$

By (5.4), (5.8), we have for $|n| \leq j_{0}$,

$$
\left|\left(H\left(x_{0}\right) R_{\mathbb{Z} \backslash\left[-j_{0}, j_{0}\right]} \xi\right)_{n}\right| \leq \sum_{\left|n^{\prime}\right|>j_{0}} e^{-\left|n-n^{\prime}\right|}\left|\xi_{n^{\prime}}\right|<e^{-\frac{M}{400}}+\sum_{\left|n^{\prime}\right|>j_{0}+\frac{M}{2}} e^{-\left|n-n^{\prime}\right|}\left|\xi_{n^{\prime}}\right|<e^{-\frac{M}{500}} .
$$

By (5.9), (5.10), we have

$$
\left\|G_{\left[-j_{0}, j_{0}\right]}\left(x_{0}, E\right)\right\|>e^{N}
$$


So if there is an extended state $\xi, \xi_{0}=1$ with energy $E$, then there is some $j_{0},\left|j_{0}\right|<N_{1}=$ $N^{C_{1}}$ ( $C_{1}$ is a sufficiently large constant), such that (by (5.11))

$$
\operatorname{dist}\left(E, \operatorname{spec} H_{\left[-j_{0}, j_{0}\right]}\left(x_{0}\right)\right)<e^{-N} .
$$

Denote $\Omega(E) \subset \mathbb{T}^{d}$ the set of $x$ such that

$$
\left|G_{[-N, N]}(E, x)(m, n)\right|<e^{N^{1-}-\frac{1}{100}|m-n| \chi_{|m-n|>\frac{N}{10}}}
$$

fails for some $|m|,|n| \leq N$. Let $\mathcal{E}_{\omega}=\bigcup_{|j| \leq N_{1}} \operatorname{spec} H_{[-j, j]}\left(x_{0}\right)$. It follows from (5.12) that if $x \notin \bigcup_{E^{\prime} \in \mathcal{E}_{\omega}} \Omega\left(E^{\prime}\right)$, then

$$
\left|G_{[-N, N]}(E, x)(m, n)\right|<e^{N^{1-}-\frac{1}{100}|m-n| \chi_{|m-n|>\frac{N}{10}}}, \quad|m|,|n| \leq N .
$$

Consider the set $S=S_{N} \subset \mathbb{T}^{d+1} \times \mathbb{R}$ of $\left(\omega, x, E^{\prime}\right)$, where

$$
\begin{gathered}
\|k \omega\|>c|k|^{-2}, \quad \forall 0<|k| \leq N, \\
x \in \Omega\left(E^{\prime}\right), \\
E^{\prime} \in \mathcal{E}_{\omega} .
\end{gathered}
$$

By (5.14), (5.15), (5.16),

$$
\operatorname{Proj}_{\mathbb{T}^{d+1}} S \text { is a semi-algebraic set of degree }<N^{C},
$$

and by Proposition 3.2

$$
\operatorname{mes}\left(\operatorname{Proj}_{\mathbb{T}^{d+1}} S\right)<e^{-\frac{1}{2} N^{\sigma}}
$$

Let $N_{2}=e^{(\log N)^{2}}$,

$$
\mathcal{B}_{N}=\left\{\omega \in \mathbb{T}\left|\left(\omega, T^{j} x_{0}\right) \in \operatorname{Proj}_{\mathbb{T}^{d+1}} S_{N}, \exists\right| j \mid \sim N_{2}\right\} .
$$

By (5.17), (5.18), (5.19), using Lemma 4.6, mes $\mathcal{B}_{N}<N_{2}^{-c}, c>0$. Let

$$
\mathcal{B}=\bigcap_{N_{0}} \bigcup_{N>N_{0}} \mathcal{B}_{N}
$$

then mes $\mathcal{B}=0$. We restrict $\omega \notin \mathcal{B}$.

If $\omega \notin \mathcal{B}_{N}$, we have for all $|j| \sim N_{2},\left(\omega, T^{j} x_{0}\right) \notin \operatorname{Proj}_{\mathbb{T}^{d+1}} S_{N}$, by (5.13),

$$
\left|G_{[j-N, j+N]}\left(E, x_{0}\right)(m, n)\right|<e^{N^{1-}-\frac{1}{100}|m-n| \chi_{|m-n|>\frac{N}{10}}} .
$$

Let $\Lambda=\bigcup_{\frac{1}{4} N_{2}<j<2 N_{2}}[j-N, j+N] \supset\left[\frac{1}{4} N_{2}, 2 N_{2}\right]$, by Lemma [5.1] we deduce from (5.21) that

$$
\left|G_{\Lambda}\left(E, x_{0}\right)(m, n)\right|<e^{-\frac{1}{200}|m-n|}, \quad|m-n|>\frac{N_{2}}{10},
$$

and therefore

$$
\left|\xi_{j}\right|<e^{-\frac{1}{1000}|j|}, \quad \frac{1}{2} N_{2} \leq j \leq N_{2}
$$

Since $\omega \notin \mathcal{B}$, by (5.20), there is some $N_{0}>0$, such that for all $N \geq N_{0}, \omega \notin \mathcal{B}_{N}$. So, (5.23) holds for $j \in \bigcup_{N \geq N_{0}}\left[\frac{1}{2} e^{(\log N)^{2}}, e^{(\log N)^{2}}\right]=\left[\frac{1}{2} e^{\left(\log N_{0}\right)^{2}}, \infty\right)$. This proves (15.6) for $j>0$, similarly for $j<0$. Hence Theorem 5.2 follows. 
Acknowledgment. The authors are very grateful to Dr. Y. Shi for the valuable suggestions. This paper was supported by National Natural Science Foundation of China (No. 11790272 and No. 11771093).

\section{REFERENCES}

[1] S. Basu. On bounding the Betti numbers and computing the Euler characteristic of semi-algebraic sets. Discrete Comput. Geom., 22(1):1-18, 1999.

[2] S. Basu, R. Pollack and M.-F Roy. On the combinatorial and algebraic complexity of quantifier elimination. J.ACM, 43(6):1002-1045, 1996.

[3] J. Bourgain. Estimates on Green's functions, localization and the quantum kicked rotor model. Ann. of Math. (2), 156(1):249-294, 2002.

[4] J. Bourgain. Green's function estimates for lattice Schrödinger operators and applications, volume 158 of Annals of Mathematics Studies. Princeton University Press, Princeton, NJ, 2005.

[5] J. Bourgain. Anderson localization for quasi-periodic lattice Schrödinger operators on $\mathbb{Z}^{d}$, $d$ arbitrary. Geom. Funct. Anal., 17(3):682-706, 2007.

[6] J. Bourgain and M. Goldstein. On nonperturbative localization with quasi-periodic potential. Ann. of Math. (2), 152(3):835-879, 2000.

[7] J. Bourgain, M. Goldstein, and W. Schlag. Anderson localization for Schrödinger operators on $\mathbb{Z}$ with potentials given by the skew-shift. Comm. Math. Phys., 220(3):583-621, 2001.

[8] J. Bourgain, M. Goldstein, and W. Schlag. Anderson localization for Schrödinger operators on $\mathbf{Z}^{2}$ with quasi-periodic potential. Acta Math., 188(1):41-86, 2002.

[9] J. Bourgain and S. Jitomirskaya. Anderson localization for the band model. In Geometric aspects of functional analysis, volume 1745 of Lecture Notes in Math., pages 67-79. Springer, Berlin, 2000.

[10] J. Bourgain and I. Kachkovskiy. Anderson localization for two interacting quasiperiodic particles. Geom. Funct. Anal., 29(1):3-43, 2019.

[11] M. Gromov. Entropy, homology and semialgebraic geometry. Séminaire Bourbaki, Vol. 1985/86 Astérisque, 145-146:225-240, 1987.

[12] W. Jian, Y. Shi and X. Yuan. Anderson localization for one-frequency quasi-periodic block operators with long-range interactions. J. Math. Phys., 60(6):063504, 15, 2019.

[13] H. Krüger. Multiscale analysis for ergodic Schrödinger operators and positivity of Lyapunov exponents. Journal d'Analyse Mathématique, 115:343-387, 2011.

(Jia Shi) School of Mathematical Sciences, Fudan University, Shanghai 200433, China

E-mail address: 15110180007@fudan.edu.cn

(Xiaoping Yuan) School of Mathematical Sciences, Fudan University, Shanghai 200433, China

E-mail address: xpyuan@fudan.edu.cn 